\title{
Was Vygotsky Right? Evaluating Learning Effects of Social Interaction in Children Internet Games
}

\author{
Franca Garzotto \\ HOC - Hypermedia Open Center \\ Department of Electronics and Information, Politecnico di Milano \\ Via Ponzio 34/5, 20133 Milano (Italy) \\ franca.garzotto@polimi.it
}

\begin{abstract}
The social basis for learning, particularly in childhood, has been acknowledged since the seminal research of the Russian psychologist Lev Vygotsky. Although his theory is very often cited in HCI literature as a theoretical basis for the design of multi-user interactive artefacts, little empirical data is available that assess Vygotsky's thesis in this domain. This paper presents an empirical study that investigated the learning impact of social interaction in the context of children online edutainment. We developed "multiplayer" and "individual" configurations of an educational internet game and measured the learning benefits of "playing together" and "playing alone" in 54 children from a local elementary school. Not surprisingly, our findings confirm that Vygotsky was right. They provide some empirical evidence that in contexts of online gaming, the presence of interpersonal communication, collective goals, and social activities has measurable beneficial effects on children learning.
\end{abstract}

Keywords: edutainment, social interaction, multiuser online game, children, learning, evaluation.

\section{Introduction}

The Russian pedagogist Lev Vygotsky (1896-1934) is one of the most cited researchers in HCI literature on social interaction and learning. The major theme of his theoretical framework is that the social dimension plays a fundamental role in the development of human cognition and learning, especially in childhood [24]. Although his work is very often invoked as a theoretical basis for the design of multi-user interactive artefacts, little data are available that empirically assess Vygotsky's thesis in situations of ICT mediated social interaction, particularly in the domain of children edutainment ${ }^{l}$.

In this paper we discuss an empirical study that investigate the learning benefits of social interaction on children engaged in internet educational games, in order to

\footnotetext{
${ }^{1}$ The term "edutainment" - standing for "educational entertainment" - denotes a form of entertainment designed to educate as well as to amuse.
} 
empirically establish whether, in this context, "playing with peers" is more conductive to learning than "playing alone", and whether different conditions of social interaction induce different measurable learning effects.

For the purpose of our study, we developed multiple configurations - multiplayer and individual - of an internet game and compared the learning effects in three groups of elementary school children using the system "alone" and "together". We also investigated the role of competition in multiuser gaming, obtaining some empirical data on how "playing against others" may affect learning.

Not surprisingly, our findings confirms Vygotsky's general thesis, highlighting that, at least in young children, internet game experiences involving social interaction are more conductive to learning than playing alone. The results of our study also show that children using the multiplayer competitive version achieved slightly better results that those using the multiplayer non-competitive version, suggesting that, in some situations, competition may foster learning.

The rest of the paper is organized as follows. We first present the game used in our research, describing its various configurations. Then we introduce the procedure adopted in our empirical study and discuss its main findings. We conclude with a brief overview of related works and some final considerations.

\section{The Online Game}

The online game used in our study is called Pirates Treasure Hunt ("Pirates" for short). It was built by our laboratory ${ }^{2}$ in the context of a larger project [9] [5] that collaborates with elementary and high schools to design, develop, and evaluate internet based edutainment experiences for children. The general educational goal of Pirates is to stimulate children interest in and attention for "other" cultures and ways of life - a subject of primary school curricula in our country. The game exposes children to contents and knowledge tasks related to the culture of two non-European countries - Morocco and Japan. To complete the game, players must apply what they have previously studied and learnt about these subjects. At a deeper level, Pirates fosters concept understanding and meaning-making, as well as recognition and recall skills.

For the purpose of our study, we developed three different versions of the game: a Multiplayer Collaborative and Competitive version (MCC Pirates), a Multiplayer Collaborative version (MC Pirates) and an Individual version (I Pirates). In the rest of this section, we introduce the general gaming rules holding in all configurations, and then describe the peculiar characteristics of each version.

\subsection{Pirates: General Rules}

Pirates is a discovery game where players act like pirates, exploring a virtual archipelago and discovering hidden "treasures". In the virtual space, a user is represented as a small ship (see figure 1 - \#3) and can navigate the archipelago either

\footnotetext{
${ }^{2}$ Using Flash Communication Server and XML technology.
} 
in a continuous way (using cursors) or "by jumps" (selecting an area on a map). The Pirates' world is populated with interactive objects - animals or environmental elements. Some of them, e.g., rocks and sharks, are "dangerous" (see figure 1 - \#2): when casually encountered or explicitly activated, they cause a penalty and a brief (10 seconds) suspension of the player's activity, during which any interaction is inhibited. Others are "treasure objects": they hide "treasures" that appear when these objects are "clicked". A treasure is a quiz card that shows an image and a quiz (see figure 2). The image represents a typical aspect of the culture of Morocco or Japan, i.e., a traditional Japanese food or a typical piece of furniture of Morocco. The quiz is a yes/no question about the image meaning, such as: "Is this Moroccan dressing?"

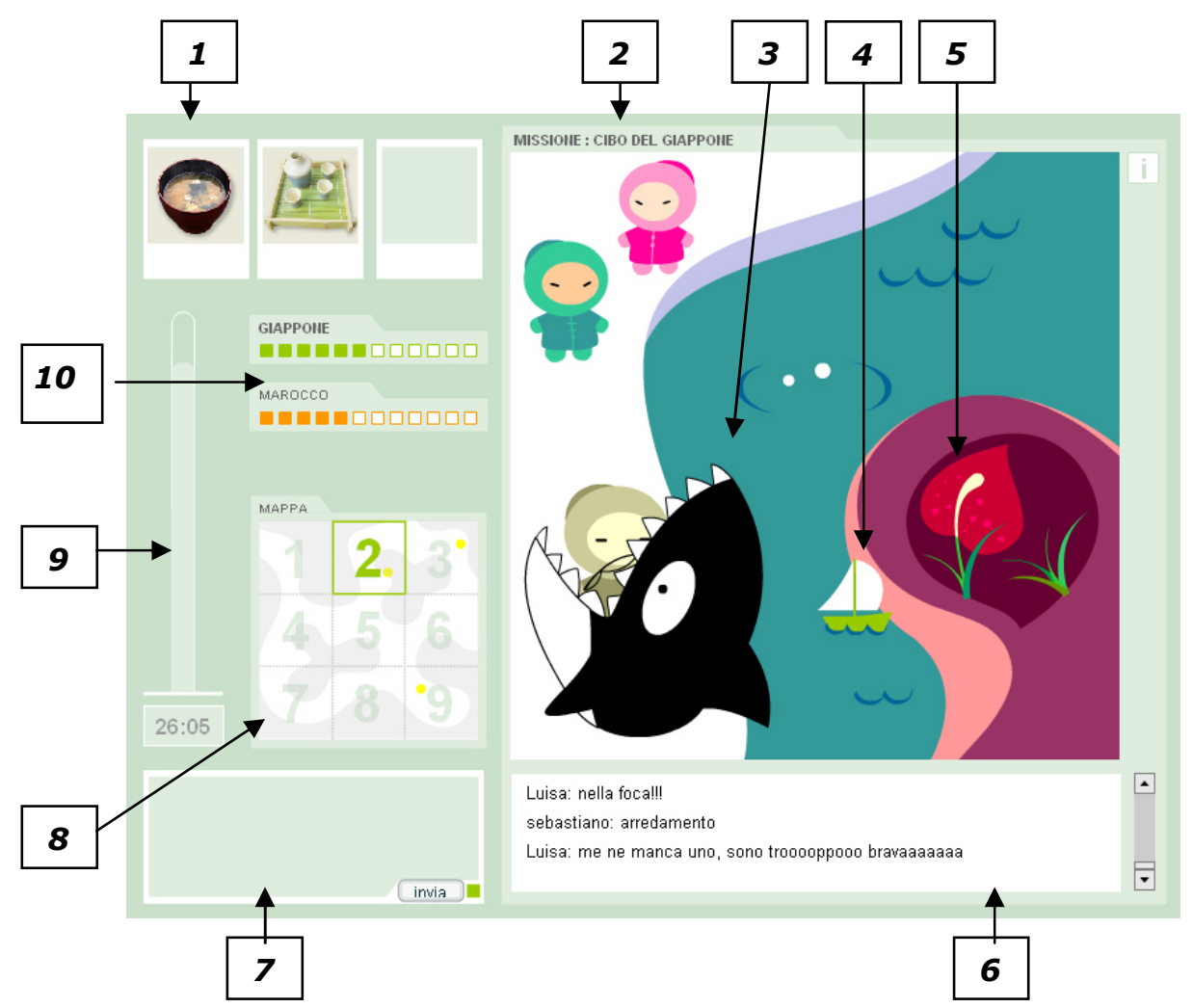

Legend:

1: Collected Treasures (Miso Soup and Sushi, in this case); 2: Player's Mission (Japanese Food in this case)

3: Dangerous Object; 4: Player's “Avatar”; 5: Treasure Object; 6: Chat Messages;

7: Chat Authoring Box; 8: Interactive Map; 9: Timer; 10: Teams' Progress Status (partially or totally removed in other configurations)

Fig. 1. Pirates interface - MCC configuration

A Pirates player has a mission: to discover and collect all treasures (i.e., cards) that concern a specific "cultural aspect" (food, dressing, tradition, or furniture) of a country. If a player's answer to a treasure question is wrong, he gets a penalty and a 
10 seconds suspension. If his answer is correct and the image corresponds to the player mission, he gains points and the card can be "picked up" (appearing on the treasure bag on the top left side of the screen - see figure 1 - \#1), contributing to the progress towards game completion.

The treasure objects hidden in the archipelago are the same in all game sessions (as discussed in section 3.2); also the images on the treasure cards are the same, but the quizzes are dynamically customized to the current mission of the player who clicks on the object and discovers the treasure. For example, a shark may hide a card showing the image of cous-cous plate but the quiz is "Is this Japanese food?" only for the player whose current mission is to collect Japanese food; another question will appear on the same card for a player who has a different mission.

\subsection{Pirates Configurations}

For the purpose of our study, we need to ensure that in all Pirates configurations players have an equivalent exposure to learning contents ${ }^{3}$ and the only aspect that is modified in the user experience concerns the social conditions in which children are exposed to such contents. To meet this requirement, in all game versions the player's tasks are the same ("explore and collect"); also the set of treasure cards hidden in the archipelago are the same, thus providing equivalent knowledge spaces to explore. We only change the rules to end the game: in all its configurations, Pirates is over when all missions for a country are completed, but in the two multiplayer modes there are both "individual" missions and a "collective mission" that must be fulfilled in order to terminate the game.

\subsubsection{Multiplayer Collaborative and Competitive Version (MCC)}

This version (see figures 1, 2 and 3) involves eight simultaneous remote players, who are organized in two competing teams, each one representing a country Morocco or Japan. A different mission is assigned to each player, but the victory is assigned to a team only if all members complete their mission and they achieve this result before the other team. In other word, the challenge is to discover and pick up all treasures (cards) for the own team country before the other team (see figure 4). This collective goal is the key motivator for collaboration and communication. A player should feel that the completion of the others' missions in his team is as important as his own mission's achievement; he is motivated to continue searching for team treasures and to offer mutual assistance even after his individual mission is completed.

The communication and collaboration tool is the chat (see figure 1 - \#7). A player can send messages to invoke or provide help, to alert the other members about pitfalls or dangerous objects in a given area, or to inform his team mates when he discovers a treasure that is relevant for the rest of the team.

\footnotetext{
${ }^{3}$ Here we use the term "content" in a broad sense, to denote two aspects: i) the information pieces that are available in the game (i.e., the treasure cards) and players get exposed to; ii) the knowledge tasks in which children get involved.
} 


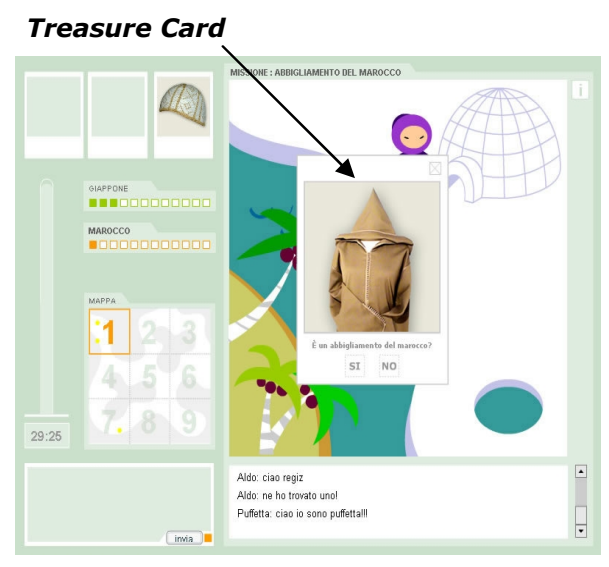

Fig. 2. MCC Configuration: treasure card

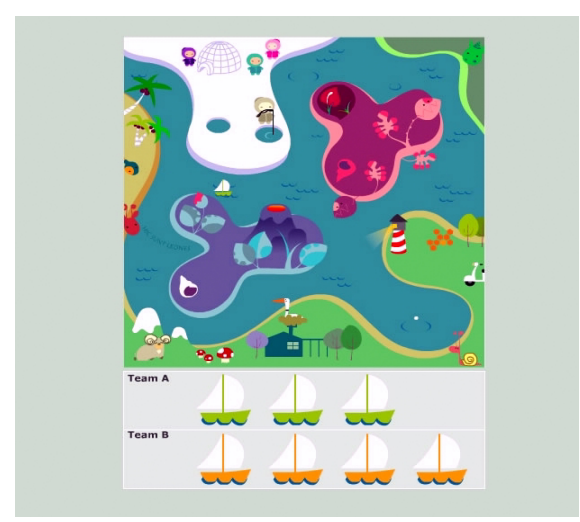

Fig. 3. MCC configuration: Players are logging-in (One player in team A - Morocco hasn't logged in yet )

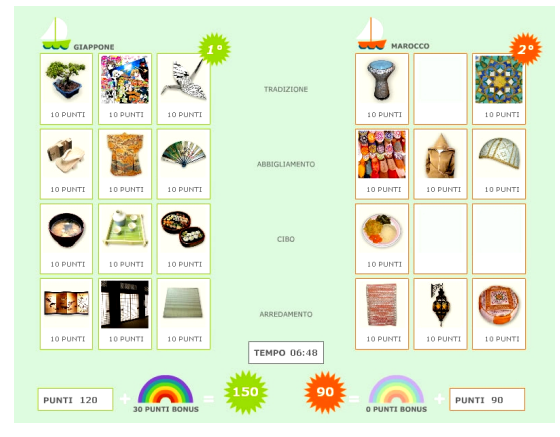

Fig. 4. End-game screen shot - MCC configuration. It shows collected treasures, gained points, penalties, and play time of each team

\subsubsection{Multiplayer Collaborative Configuration (MC)}

In this version, four remote children play together for the same country. The interface is essentially the same as in the MCC version, but the progress bar now presents the progresses of one country only (see figure 5), and the end-game screen displays the final results for that country only. In absence of competition, what are the motivations for social interaction? One motivation could be enjoyment for "getting in touch" with remote peers (via chat) and feeling part of a community. Or a child may be in trouble and may need to ask the others for help. Or he may contribute to other's missions for the purpose of speeding up the game conclusion: minimizing the session duration is a form of challenge - a kind of self-competition at group level, and a selfrewarding feature per se. 


\subsubsection{Individual Player Configuration (I)}

In the individual version, the player is initially assigned to a country and a mission (as in the multiplayer versions), and a new mission for the same country is automatically assigned after the current mission is completed. As in the MC version, the game terminates when all treasures for the current country have been discovered.

In the individual configuration, the chat has the role of personal "notebook", where the user can take notes and record information that can be useful later, e.g., annotating where he find the dangerous objects or the locations where treasures for future missions can be found.

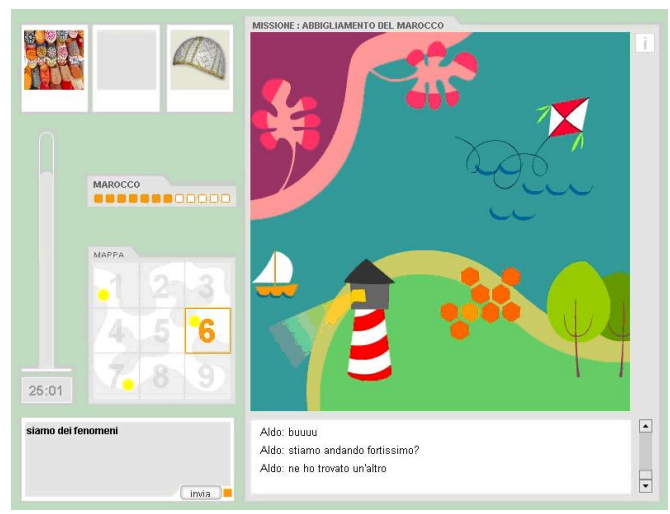

Fig. 5. Pirate's interface - MC configuration

\subsection{Educational Support Material}

In order to properly answer treasure quizzes, children must have previously acquired some "domain knowledge" about the game subjects. For this purpose, we provided some printed educational material and integrated the game with an online, individual, 3D learning space. Both are intended to be used before gaming. The printed material, which can be studied in the classroom or at home before gaming (see next section),
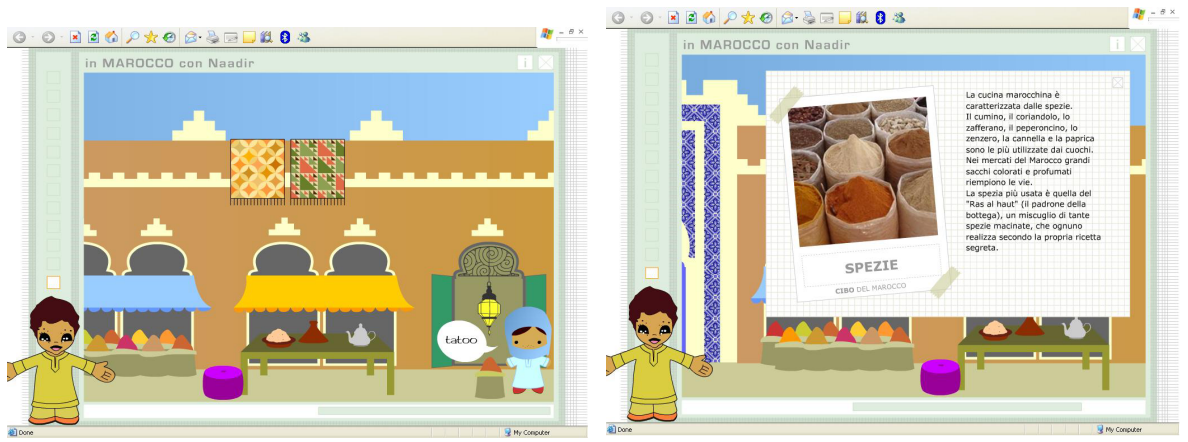

Fig. 6. The 3D learning space 
comprises information sheets about the various cultural topics of the game, including images and texts describing the subjects of the treasures hidden in the game.

The online learning space (see figure 6) provides two cartoon-like 3D worlds that reproduce "typical" environments of Morocco or Japan (e.g., a Japanese house or a Moroccan "suk"); these virtual spaces include a number of interactive objects that show "in context" the game cultural elements of each country (examples of food, dressing, traditions, furniture that represent the game treasures) . When an interactive element is selected, the corresponding information sheet appears (see figure 6- right). The printed material and the information sheets in the learning environment both use the same images as the treasure cards in the game.

\section{Evaluation Procedure}

The general goal of the study was to assess empirically two hypotheses:

i) social interaction in online edutainment promotes children's learning; in particular, playing an online educational game together is more effective for learning than playing alone;

ii) different conditions of social interaction induce different learning effects.

For the purpose of our study, we measured and compared some learning results achieved by three groups of elementary school children who used Pirates in the different configurations discussed in the previous section. We considered learning benefits at two levels of Bloom's taxonomy of the learning domain [1]: the cognitive level (which involves knowledge and intellectual skills) and the affective level (which involves the manner in which learners deal with things emotionally - e.g., feelings, values, appreciation, enthusiasms, motivations, and attitudes). At the cognitive level, we focused on the cognitive skills of recall, recognition, and understanding of specific facts or concepts. At the attitude level, we considered motivation to learning new subjects and interest to "other cultures and ways of life", which are the subject of the educational content of Pirates.

The study involved 54 children aged 8-9 (28 girls and 26 boys), from three third grade classes of a public elementary school in our city. All children had a sufficient level of skills in the use of mouse and keyboard, and a homogenous exposure to interactive experiences. We randomly organized participants in three groups, one per game configuration, composed of 16 children. Children played with the game in the two computer laboratories of the school, which is the conventional context where children in our country use computers in their normal school activity. When using the multiplayer configurations of Pirates, we simulated the effect of playing with "remote" peers by allocating children of the same team in different computer labs. Rooms were quite wide, and children in the same lab were placed far enough so that they could not peep nor could be distracted by what the others in the same space were doing. Six teachers (two per class) were also involved in the study; they offered assistance during the play sessions and were interviewed afterwards.

Each group playing a multiplayer configuration (MMC or MC) was split in four subgroups, each one composed of four randomly selected children. Subgroups 
Table 1. Children' allocation to game configuration in the laboratory sessions (MCC=Multiplayer Collaborative Competitive; $\mathrm{MC}=$ Multiplayer Collaborative; I = Individual)

\begin{tabular}{|c|c|c|c|}
\hline Lab Session \# & Configuration & Kids & Sub-groups \\
\hline $\mathbf{1}$ & MCC & $\mathbf{8}$ & $\mathbf{2}$ \\
\hline $\mathbf{2}$ & MCC & $\mathbf{8}$ & $\mathbf{2}$ \\
\hline 3 & MC & $\mathbf{4}$ & $\mathbf{1}$ \\
\hline $\mathbf{4}$ & MC & $\mathbf{4}$ & $\mathbf{1}$ \\
\hline $\mathbf{5}$ & MC & $\mathbf{4}$ & $\mathbf{1}$ \\
\hline $\mathbf{6}$ & MC & $\mathbf{4}$ & $\mathbf{1}$ \\
\hline $\mathbf{7}$ & I & $\mathbf{1 6}$ & n.a. \\
\hline
\end{tabular}

playing the competitive configuration were paired to play one against the other. We conducted seven laboratory sessions with children, of the average duration of 2 hours, involving children and sub-groups as described in the following table.

For all groups, the work was organized in three phases:

Phase 1 - Study and learning pre-test. Some days before a laboratory session, children studied and discussed the printed material in the classroom for one hour, and spent approximately 45 minutes using the 3D learning space of Pirates with their teachers. This activity aimed at supporting a homogeneous level of domain knowledge in all children in our study. At the end of this work, we submitted a learning test to assess children learning level on game subjects. Knowledge and intellectual skills (recognition, recall, concept understanding) were operationalized in terms of children' capability to assign meaning to images. The test included ten "quizzes". A quiz was a checklist question. It showed an image of the culture of Morocco or Japan (e.g., a Moroccan dress) randomly selected from the set of the game treasure cards, asking "what is this..?"; the four alternative answers were defined in terms of country and cultural type aspect, e.g., "Japanese Food".

Phase 2 - Play. Before playing the game, children were trained about game rules and spent approximately 10 minutes to practice with interaction commands, on a demo-version of Pirates. Finally, they played the real game twice, each time changing country and initial mission (see figure 7 - left side).

Phase3 - Learning post test and questionnaire. After gaming, we submitted a second learning test, similar to the first one but with a different set of quizzes. In addition, children filled a simple questionnaire (see figure 7 - right side) aimed at giving us some quantitative and qualitative information about learning benefits at the affective level, and about satisfaction and enjoyment. The questionnaire included few open and close ended questions, asking children how much they liked the gaming experience, what they liked and disliked more, and similar. A suggested in [19], we

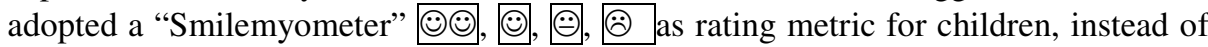
numerical scores.

Beside learning tests and questionnaires, we collected qualitative data by observing children behavior and by interviewing the teachers. During play, an observer sat sideby-side each child and took notes about his behavior (voice comments, movements, strategies, interaction mistakes, focus of attention,...) on a structured evaluation form. Interviews with teachers were held some days after the test sessions, and mainly attempted to get insights about learning benefits at attitude level. 


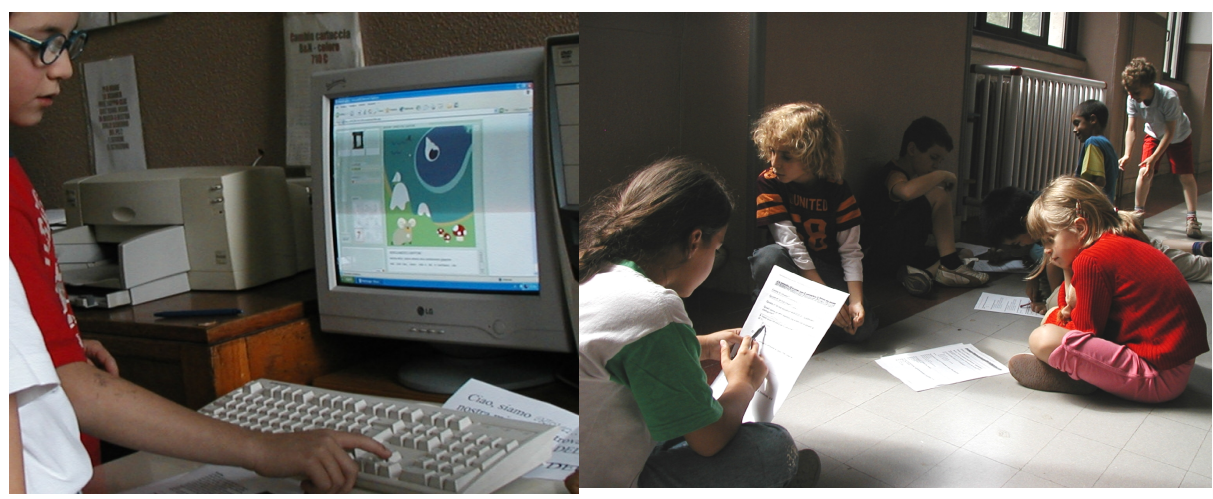

Fig. 7. Playing Pirates (left) and filling the questionnaire (right)

\section{Findings}

The main measure for learning at the knowledge level was the difference in the scores (number of correct answers) of pre and post test learning tests. The average difference of correct questions in the three groups (MMC, MC, I) is shown in fig. 8, that highlights that in all configurations the gaming experience induced some learning benefits at knowledge level.

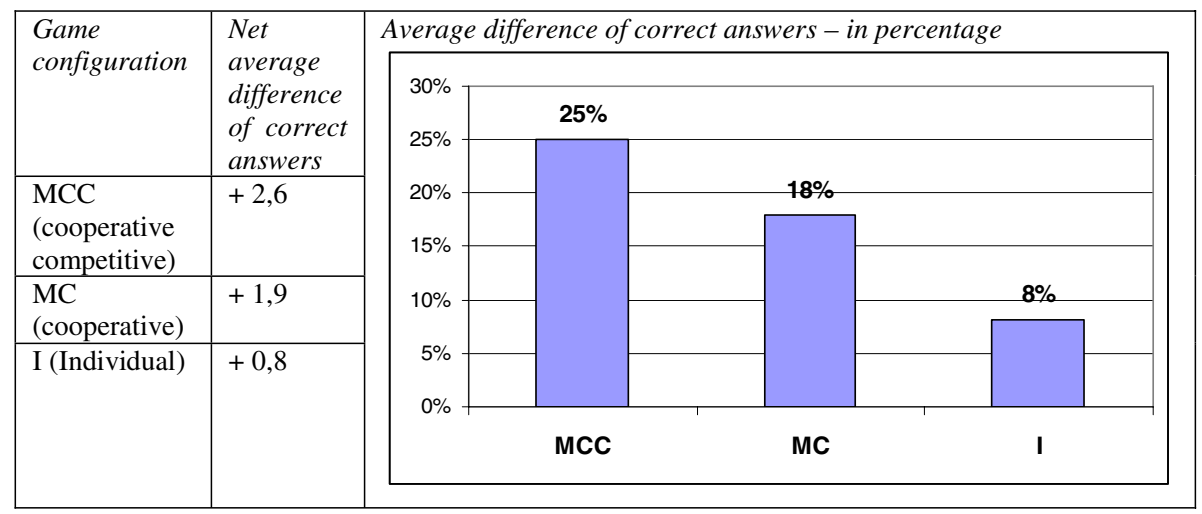

Fig. 8. Measuring Learning Benefits at Knowledge level: means of score differences in pre-post learning tests

To measure the learning benefits at the affective level (motivation to learning new subjects and interest to "other" cultures and ways of life) we analyzed various sources: quantitative and qualitative results of children' questionnaire, teachers' answers during interviews, and observers' notes. In the questionnaire, for example,

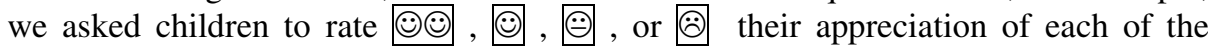




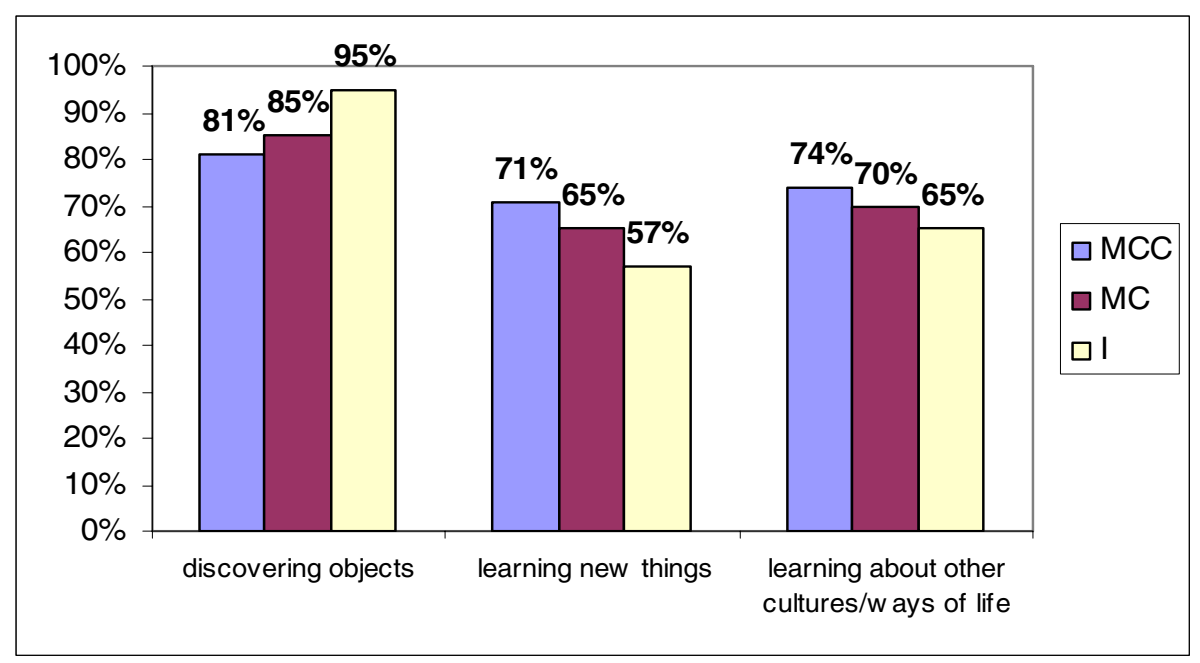

Fig. 9. Children' $;$-; ratings for question "How much did you like...?"

other countries and ways of life”. Figure 9 visualizes the percentages of children in the three groups who rated $;-;$ a specific aspect. It shows that the discovery features of the game is the aspect that children appreciated more - this is, indeed, the truly "gaming" aspect of Pirates' experience, common to all configurations.

The fact that in all three groups more that $50 \%$ of children gave the highest possible rate also to "Discovering new things" and "Learning about other cultures and ways of life", highlights that the overall experience fostered positive feelings towards the educational content of the game. Still, the number of children who rated learning aspects as $\odot$;) was larger in those playing together (the MCC and MC groups) than in those playing alone (I group). These data may be interpreted as a positive result achieved at affective level by the MMC and MC groups. At least, they reveal that children playing in the two contexts of social gaming became more self-conscious of the fact that they learned something new, or were more willing to acknowledge this aspect as positive, than their peers playing alone. This affective aspect seems slightly stronger in presence of competition (MCC group).

Qualitative data analysis partially confirms these results. We coded the "free notes" reported in children questionnaires and their voice comments transcripted by the observers. Then we clustered the contributions denoting positive feeling towards "learning new things" or "learning about other countries and ways of life. (Examples of children' comments in this cluster are: 'I didn't' know that my favorite cartoons are from Japan! It's great! Now I understand why characters always eat that funny rice!"(written comment); "Look at origami! Do children in Japan build origami at school as we do?"(voice comment) ${ }^{4}$. The number of contributions that externalized

${ }^{4}$ In the above comments, children refer to "manga" comics, sushi, and origami, appearing in Pirates as treasures for missions related to Japan. Origami is an activity that some of our children practiced at school. 
positive feelings about learning was higher in the groups playing in a social mode than in the individual mode $(50 \%$ and $35 \%$ more in the MCC and MC groups respectively, than in the I group). Still, at the affective level no significant difference among the three groups was reported by teachers. Teachers involved children in classroom and home "conventional" activities on the game subjects (discussion, storytelling, drawing). In all participants they noticed interest and enthusiasm for such topics, which were totally new for most students, and do not observed any significant difference in the three groups.

As a further input for analysis and reflection, we considered enjoyment. We analyzed the scores given to the questionnaire question: "How much did you enjoy playing Pirates? $\odot-;)(-)$. As shown in the following diagram, more people in the MCC group (75\%) rated enjoyment very high $\odot-;$, against the $64 \%$ of MC group and the $41 \%$ of I group.

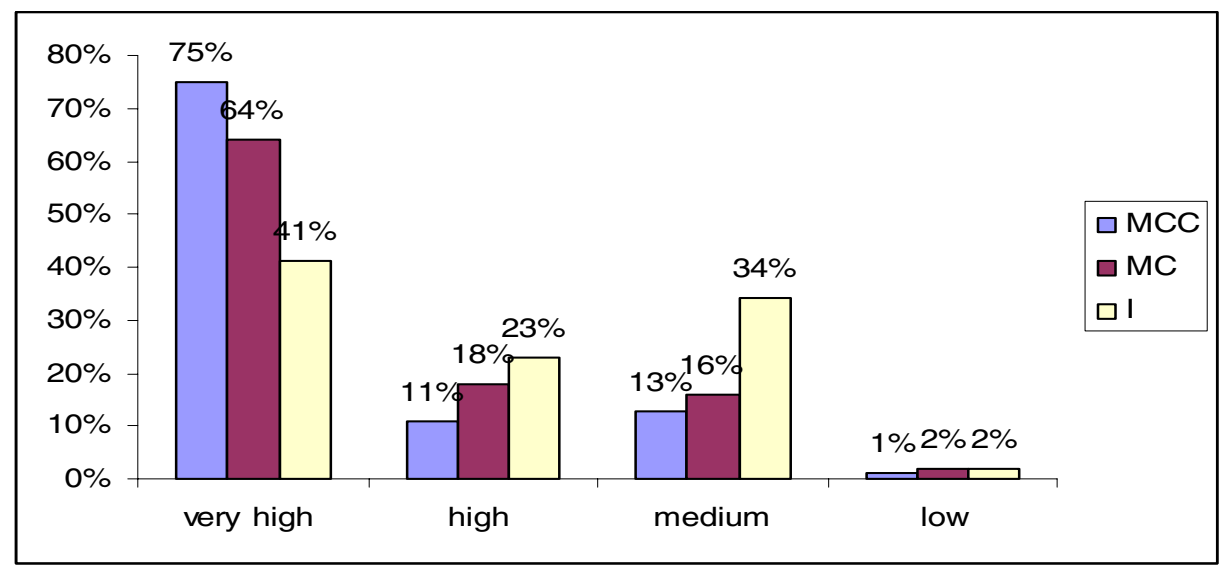

Fig. 10. Percentages of children' high rating $\odot$;) for enjoyment

The analysis of qualitative data confirmed this trend. We considered the number of comments in the questionnaire, transcripts of voice comments, and observers' notes about children' behaviour during the game that denoted high degree of enthusiasm, appreciation, or engagement (e.g., "I would like to do Pirates thousands times!!!", "I wish I had Pirates at home thus I could play it every day!"'). The number of contributions coded as "enjoyment" resulted higher in the MCC group than in the MC group, who in turn showed more enjoyment than the I group. For example, in the MCC group the progress bar was wept franticly under control (more constantly than the other groups), and any progress of the own team was outlined by enthusiastic comments. The happiness of victorious children was enormous (they shouted and jumped for 5 minutes).

\section{Discussion}

As we expected, the findings of our study provide an empirical validation of Vygotsky's general thesis in the context of internet edutainment: in young children, 
online game experiences involving social interaction are more conductive to learning than playing alone. We measured stronger educational benefits in social gaming w.r.t. individual play at both the knowledge and the attitude levels of Bloom's taxonomy. In this section, we attempt an interpretative analysis of our findings. In our case study, social interaction took three forms: communication, remote cooperation, and competition.

Communication, enabled by the chat, created the feeling of "being there with many" ("co-presence" [14]) and of being "a member of a group" ("community" [26]). The "experience of connection" enhanced the sense of social identity and enforced the feeling of self-recognition without which learning attitudes, in children, may be weakened [21]. Discussing strategies and solutions with others was a way "to make thinking visible" and created a more tangible context for what had been learned, building the conditions for situated learning [12].

Remote cooperation with distant peers (implicit in the concept of "multiplayer online" game) was founded on shared goals, which pinpointed in children the need for more than one person to be involved and for complementary abilities, and increased their motivation for applying individual skills and knowledge.

Competition was a powerful motivator for being engaged in educational content. Children gained satisfaction from competing against and beating other people; competition added emotions that made the gaming experience more exciting and fun [4][13][15][23][25] - all aspects that fostered learning.

The results of our study also support our second hypothesis, i.e., different conditions of social interaction induce different learning effects. Our data show some correlation among learning and intensity of social interaction. As observed in the previous subsection, communication and collaboration among remote peers was more intense in the MCC group, which apparently achieved stronger learning benefits than in the $\mathrm{MC}$, and became stronger towards the end of the game. Higher intensity of social interaction may be induced, in our case, by the only two aspects in which the MCC and MC configurations differ: the number of players involved (8 against 4 ) and the competitive nature of the MCC game experience. Although competition is in antithesis of cooperation, the need or desire to compete with others is a very common impetus that motivates individuals to cooperate with each other in order to form a stronger competitive force. After completing their individual mission, MCC children showed a more intense desire to continue playing if compared to the MC group, and cooperation and communication became more intense - they wanted to win!

Our study also provides some empirical evidence of some other emotional factors that are induced by social interaction and foster learning. A correlation that our data pinpoints (see figure 10) is between learning and enjoyment, showing that children who learnt more (the MCC group) also had more fun. The relationship among these two factors has been largely investigated [18]. In our case, the main variable that seemed to affect enjoyment (and, through enjoyment, increased learning) is competition. In this respect, our results differ from other studies (e.g., [17]) that found that the competitive nature of a social experience may hamper reflection and wellthough decision making, and induce less learning benefits that a non competitive version of the same experience. 


\section{Related Works and Conclusions}

The combination of social interaction and gaming was investigated by Vygotsky himself: although he is less known for his research on play, he studied children gaming as a psychological phenomenon and acknowledged its role in the child's development, both in individual and social contexts [24].

Since the advent of multimedia technology, the educational potential of multiplayer interactive games has been acknowledged by many empirical studies in HCI [3][7][10][11][20][22] and has its theoretical foundation in a number of classical and modern theories in cognitive science and pedagogy [21][26]. Still, relatively few empirical studies exist that investigate the educational benefits of social online gaming for young children. Most of $\mathrm{HCI}$ empirical research on online multiplayer games scarcely addresses this target [22], mainly focusing on adults [6][11] or adolescents [5] (and marginally considering educational aspects). The HCI literature reports a number of empirical results on social playing and learning for pre-school or primary school children, but most of the published studies exploit "complex" technological settings for the social gaming experience (tabletops, tangibles, robots, mobile devices, augmented reality systems, etc.) [3][7][16]; apparently, the main goal of these studies is more to test the design of novel forms of interactions than to evaluate how the social dimension of these innovative solutions may affect learning.

The main contribution of our work is to empirically investigate game-based learning [18] and social interaction in young children and in a conventional technological domain - the Internet - that is becoming more and more popular in education, both in conventional settings and in home-schooling situations.

Acknowledgments. The author is grateful to the technical staff of HOC Lab. Special thanks go to the children and the teachers of elementary school "Nolli Arquati" in Milan who participated so enthusiastically in the evaluation study.

\section{References}

1. Bloom, B.S., Mesia B.B., Krathwohl D.R.: Taxonomy of Educational Objectives New York. David McKay., (1964)

2. Csikszentmihalyi, M.: Flow: The Psychology of Optimal Experience. Harper Perennial, New York (1991)

3. Deguchi, A., et al.: A system for supporting childrens face-to-face collaborative learning by integrating personal and shared spaces. In: Proc. IDC'2006, Tampere, Finland, ACM Press, New York (2006)

4. Desurvire, H., Maplan, M., Toth, J.A.: Using heuristics to evaluate the playability of games. In: Extended Abstracts of CHI 2004, Vienna, pp. 1509-1512. ACM Press, New York (2006)

5. Di Blas, N., Paolini, P., Poggi, C.: Educational benefits: testing and evaluation of a collaborative 3d world. In: Proc. ED-MEDIA 2005, AACE, pp. 1002-1011 (2005)

6. Ducheneaut, N., Yee, N., Nickel, E., Moore, R.: Alone Together Exploring the social dynamics of massively multiplayer online games. In: Proc. CHI2006, pp. 407-416. ACM Press, New York (2006)

7. Druin, A. (ed.) The Design of Children Technology. San Francisco (CA) (1999) 
8. Fisch, M.S.: Making Educational Computer Games “Educational”. In: Proc. IDC'05, Boulder (Colorado), ACM Press, New York (2005)

9. Garzotto, F., Forfori, M.: FaTe2: Storytelling Edutainment Experiences in 2D and 3D Collaborative Spaces. In: Proc. IDC '06, Tampere (Finland), ACM Press, New York (2006)

10. Hanna, L., Neapolitan, D., Risden, K.: Evaluating Computer Game Concepts with Children. In: Proc. IDC 2004, College Park (Maryland), pp. 49-56. ACM Press, New York (2004)

11. Nardi, B., Harris, J.: Strangers and Friends: Collaborative Play in World of WarCraft. In: Proc. CSCW'06, Banff (Canada), pp. 1-10. ACM Press, New York (2006)

12. Lave, J., Wenger, E.: Situated Learning: Legitimate Peripheral Participation. Cambridge University Press, Cambridge (1990)

13. Lazzaro, N., Keeker, K.: What's my method? A game show on games. In: Extended Abstracts of CHI 2004, Vienna, pp. 1093-1094. ACM Press, New York (2006)

14. MacIntyre, B., Bolter, J.D., Gandy, M.: Presence and the aura of meaningful places. In: Proc. Presence 2004, Valencia (October 2004)

15. Pagulayan, R., Keeker, K., Wixon, D., Romero, R., Fuller, T.: User-centered design in games. In: Jacko, J.A., Sears, A. (eds.) The HCI Handbook, pp. 883-905. Lawrence Erlbaum Associates, Mahwah (2003)

16. Piper, A.M., O'Brien, E., Morris, M.R., Winograd, T.: SIDES: A Cooperative Tabletop Computer Game for Social Skill Development. In: Proc. CSCW'06, Banff (Canada), pp. 1-10. ACM Press, New York (2006)

17. Pal, J., Pawar, U., Brewer, E., Toayama, K.: The case for multiuser design for computer aided learning in developing regions. In: Proc. WWW 2006, pp. 781-789. ACM Press, New York (2006)

18. Prensky, M.: Digital Game-Based Learning. McGraw-Hill, New York (2001)

19. Read, J.C., MacFarlane, S.J.: Using the Fun Toolkit and Other Survey Methods to Gather Opinions in Child Computer Interaction. In: Proc. IDC'2006, Tampere, Finland, ACM Press, New York (2006)

20. Revelle, G.: Educating via Entertainment Media: the Sesame Workshop approach. ACM Computer and Entertainment 1(1), 1-9 (2003)

21. Rogoff, T.: Apprenticeship in Thinking: Cognitive development in social context. Oxford University Press, Oxford (1990)

22. Steiner, B., Kaplan, N., Moulthrop, S.: When play works: Turning game-playing into learning. In: Proc. IDC'2006, Tampere, Finland, ACM Press, New York (2006)

23. Sweetser, P., Wyeth, P.: Game Flow: A Model for Evaluating Player Enjoyment in Games. ACM Computer and Entertainment 3(3), 1-23 (2005)

24. Vygotsky, L.S.: Mind and Society: The development of higher psychological Process. In: Cole, M., John Steiner, V., Scribner, S., Souberman, E. (eds.), Harvard University Press, Cambridge (1978)

25. Vorderer, P., Hartmann, T., Klimmt, C.: Explaining the enjoyment of playing video games: The role of competition. In: Proc. of the 2nd International Conference on Computer Games (Pittsburgh, PA) (2003)

26. Werger, E.: Communities of Practice, Learning, Meaning, and Identity. University Cambridge Press, Cambridge (1999) 